\title{
The use of qualitative approach to detect and assess potential drug-drug interactions which may decrease the effectiveness of oral contraceptives - pilot study and literature review
}

\author{
Karolina Kilińska', Magdalena Cerbin-Koczorowska', Arleta Matschay', \\ Michał Mierzwicki', Karolina Chmaj-Wierzchowska ${ }^{2}$ \\ ${ }^{1}$ Department of Pharmaceutical Technology, Pharmacy Practice Division, Poznan University of Medical Sciences, Poland \\ ${ }^{2}$ Department of Mother and Child Health, Poznan University of Medical Sciences, Poland
}

\begin{abstract}
Aim. The aim of the pilot study was to assess the effectiveness of the tool designed for detecting potential drug-drug interactions of combined oral contraceptives (COCs) with particular emphasis on those which can affect their contraceptive action. A proper study protocol design seems to be essential for further analysis of more data and for establishing correlations between observed interactions and demographic variables.

Material and methods. The cross-sectional descriptive, retrospective study was carried out on Polish females from March to May 2013. Gathered data, including products used concomitantly with contraceptive drugs, were derived electronically by patients and underwent thematic analysis.

Results. Out of 49 respondents who agreed to participate in the study and fit the inclusion criteria only 15 derived qualitative data about other medicinal products they used. However, some of them sent their monthly report more than once, which gave the total of 158 drugs listed in 25 forms gathered during the whole pilot study. Fifty-three potential drug interactions were found, including $13(24.53 \%)$ which could have decreased the effectiveness of contraceptive drugs.

Conclusions. Continuation of the study in accordance with the study protocol will result in identification of common potential drug-related problems, which may enable development of an educational solution for gynecologists, pharmacists and patients increasing their awareness of the potential risk of contraceptive failures and unintended pregnancies.
\end{abstract}

Keywords: qualitative approach, combined oral contraceptives, drug interactions, contraceptive failures.

\section{Introduction}

Combined oral contraceptives (COCs) are one of the most popular methods of preventing unintended pregnancy $[1,2]$. Women choose COCs for their easy use, reversibility of fertility, wide access and safety [3-5]. Another important matter is their high effectiveness described with the Pearl index. This ratio was first described by Raymond Pearl in 1933 to present the number of 'failures' observed in groups of contracep- tion users in a one-year time period [6]. Two Pearl indexes are usually defined: one for 'normal use' (contains all pregnancies during the whole time of exposure) and another for 'perfect use' (includes only the pregnancies which occurred during correct and consistent use). For COC use, those ratios are estimated at 8 and 0.3 , respectively [7]. Differences in these two values may result from many factors, such as inconsistent use or concomitant use of other drugs. A list of 
these substances is very long and includes drugs for a short-term treatment as well as medicines for chronic diseases, and those which are frequent ingredients of commonly used dietary supplements [8]. For this reason special attention should be paid to ensure safety and effectiveness of patients' self-treatment.

\section{Aim}

The main study was designed to (i) identify potential drug-drug interactions of COCs, (ii) determine those which can cause a decrease in their effectiveness, and (iii) describe relationships between interactions and different demographic variables, such as age or education level. However, prior to the quantitative analysis, a pilot study was carried out to assess the effectiveness of the designed tool. This publication was prepared to present main results of the initial qualitative assessment and discuss the need for further studies in this area.

\section{Material and methods}

Study setting and sample

At the very beginning of the study (December 2012), a paper version of the questionnaire was developed. Four randomly chosen community pharmacies were invited to cooperate in the study. Pharmacy employees were asked to administer the questionnaire with information about the survey directly to those female patients who bought oral contraceptives. To assure respondents' anonymity, an envelope was attached to each questionnaire and a box for filled questionnaires was provided in the pharmacy. Only 23 questionnaires (30.67\%) out of 75 prepared ones were distributed to patients and only 3 were filled in and returned, which gave the response rate of $13.04 \%$. That is why the study protocol was revised, and an online method of data collection was designed for the second stage. The appropriate pilot study was carried out from March to May 2013. The sample consisted of females who declared use of oral hormonal preparations for their contraceptive action. Other characteristics, such as age or education level, were used to differentiate respondents within the group. The both study protocols were approved by the Ethical Committee of the Poznan University of Medical Sciences (Poland).

\section{Data collection and analysis}

The protocol of the cross-sectional descriptive study involved the use of two questionnaires. Questionnaire A collated sociodemographics and questions related to patients' general health, contraceptive behaviors and sources of knowledge of contraceptive pills. Questionnaire B was designed for respondents to fill in all the products (including drugs, dietary supplements and herbs) which they took during the last month. Prior to the collection of primary data, the literature review was conducted by performing several searches of literature indexed in MEDLINE (PubMed), Web of Science and Google Scholar, to gather information about different types of COC interactions and their clinical significance. Subsquently, data gathered with the use of Questionnaire B underwent thematic analysis and were compiled with those collected during the desk research. Although this pilot study was aimed at assessment of the adequacy of the designed tool and providing qualitative data, collection of a greater number of questionnaires will enable performing also a quantitative analysis and checking the correlation between interactions and different variables, such as education level or number of drugs taken at the same time.

\section{Results}

During the 3-month time period of the pilot study, 25 questionnaires B were collected (Table 1). However, it should be highlighted that the aim of qualitative

Table 1. General analysis of received Questionnaires B

\begin{tabular}{|c|c|c|c|c|}
\hline \multirow{2}{*}{ COC components } & \multirow{2}{*}{ Collected questionnaires } & \multirow{2}{*}{ Drugs $^{\text {a }}$} & \multicolumn{2}{|c|}{ Potential drug-drug interactions } \\
\hline & & & total & affecting COC effectiveness $^{b}$ \\
\hline $\mathrm{EE}^{\mathrm{c}}$, Cyproteronum & 2 & 5 & 3 & 1 \\
\hline EE, Desogestrelum & 6 & 36 & 5 & 0 \\
\hline EE, Dienogestum & 3 & 34 & 10 & 3 \\
\hline EE, Drospirenonum & 7 & 42 & 16 & 3 \\
\hline $\mathrm{EE}$, Gestodenum & 5 & 36 & 17 & 6 \\
\hline EE, Norgestimatum & 2 & 5 & 2 & 0 \\
\hline Total & 25 & 158 & 53 & 13 \\
\hline
\end{tabular}

${ }^{a}$ number of products listed in the questionnaire, including drugs, dietary supplements and herbs, without $\mathrm{COC}$

${ }^{b}$ number of drug-drug interactions which can decrease the COC effectiveness

'ethinyl estradiol 
research was to develop concepts rather than to verify existing theory, that is why statistical representativeness was not considered as a prime requirement [9] and so the number of gathered forms was considered to be sufficient for proper course of this pilot study. Analysis of all potential drug interactions between oral contraceptives and other substances (including drugs, dietary supplements and herbs) was made, focused on those that can decrease the COC effectiveness. A total of 158 drugs was listed in received 25 questionnaires (mean of 6 drugs per questionnaire; range 1-17). Among a total of fifty-three potential drug interactions found (33.54\%), 13 are thought to be able to decrease the effectiveness of contraceptive pills (Table 2). Another 40 interactions are thought to not affect the COC effectiveness, although they can have other undesirable effects (such as breakthrough bleeding or hyperkalemia) or decrease the effectiveness of treatment of coexisting diseases.

\section{Discussion}

The concentration of contraceptive hormones in blood may change due to concomitant use of other drugs. On the other hand, contraceptive hormones may increase or decrease the serum level of other drugs. As mentioned, women using oral contraceptives should be careful about the selection of therapeutic agents (medicines, herbs, dietary supplements), especially when there is a risk of contraceptive failure or other adverse effects [29]. It is also important that each healthcare practitioner involved in designing therapeutic recommendations should have knowledge of other substances taken by the patient.

\section{Pilot study protocol and its limitations}

Since potential drug-related problems were detected during the pilot study, the designed tool was considered suitable for identification of drug-drug interactions. However, the study protocol had also a few limitations. First of all, identification of potential interactions did not provide information about their true clinical and/or toxicological consequences. Additionally, even though several potential interactions were detected, investigators could not have reacted in time because (i) the data had been gathered retrospectively, and (ii) investigators had not obtained patients' consents to discuss findings with a respective medical practitioner. That is why another study design, which will take into account ongoing cooperation between a physician, a pharmacist and a patient, is needed. Finally, high differentiation of COC components and the small number of received questionnaires need to be highlighted. Both of these made a valuable statistical analysis impossible. Although pilot study sample seems to be large enough to enable the qualitative exploration of different types of potential drug interactions with $\mathrm{COC}$ and reasons of their possible influence increasing risk of contraceptive failures, still obtaining some quantitative results requires gathering and analysis of more plentiful data. Further exploration of data gathered with the use of both Questionnaires $A$ and $B$

Table 2. Potential drug-drug interactions identified during the study that can decrease the effectiveness of combined oral contraceptives

\begin{tabular}{|c|c|c|c|c|}
\hline COC components & Other substances & $\begin{array}{l}\text { No. of cases } \\
\text { (no. of patients) }\end{array}$ & Pharmacological effect & Potential therapeutic outcomes \\
\hline EE*, Gestodenum & spiramycinum & $1(1)$ & \multirow{4}{*}{$\begin{array}{l}\text { changes in EE enterohepatic circulation } \\
\qquad[10-13]\end{array}$} & \multirow{4}{*}{$\begin{array}{l}\text { decrease in the COC effectiveness in case of } \\
\text { high sensitivity of the intestinal flora to } \\
\text { antibiotics; diarrhea or vomiting during } \\
\text { antibiotic therapy [10-16] }\end{array}$} \\
\hline EE, Gestodenum & norfloxacinum & $1(1)$ & & \\
\hline EE, Drospirenonum & clarithromycinum & $1(1)$ & & \\
\hline EE, Drospirenonum & azithromycinum & $1(1)$ & & \\
\hline EE, Cyproteronum & Hypericum herba & $1(1)$ & $\begin{array}{l}\text { low doses - no changes in COC } \\
\text { pharmacokinetics [17]; } \\
\text { high doses - induction of CYP3A4 and } \\
\text { P-glycoprotein transporter; effect on the } \\
\text { metabolism of steroid hormones [18-22] }\end{array}$ & $\begin{array}{c}\text { low doses - lack of adverse } \\
\text { drug reactions [17]; } \\
\text { high doses - breakthrough bleeding, } \\
\text { changes in bleeding time [18-22] }\end{array}$ \\
\hline $\mathrm{EE}$, Gestodenum & $\begin{array}{l}\text { Sennae folium } \\
\text { Aloe capensis } \\
\text { Frangulae cortex }\end{array}$ & $3(1)$ & $\begin{array}{c}\text { decrease in EE absorption and shorter } \\
\text { time of detectable COC levels in blood } \\
{[23,24]}\end{array}$ & lower COC effectiveness $[23,24]$ \\
\hline EE, Dienogestum & \multirow[t]{2}{*}{ dietary fiber } & $3(1)$ & \multirow{2}{*}{$\begin{array}{l}\text { decrease in EE absorption resulting in a } \\
\text { lower EE serum concentration; inhibition } \\
\text { of enterohepatic circulation; accelerated } \\
\text { COC excretion [25-27] }\end{array}$} & \multirow{2}{*}{ lower COC effectiveness [25-27] } \\
\hline $\mathrm{EE}$, Gestodenum & & $1(1)$ & & \\
\hline $\mathrm{EE}$, Drospirenonum & carbo medicinalis & $1(1)$ & $\begin{array}{l}\text { COC adsorption, interrupted EE } \\
\text { enterohepatic circulation, decrease in } \\
\text { the EE concentration in blood [28] }\end{array}$ & lower COC effectiveness [28] \\
\hline
\end{tabular}

* ethinyl estradiol 
is thought to be essential in order to form the basis for more complex findings such as establishing correlations between observed interactions and different variables like age, education level, comorbidities, duration of COC use or even sources of knowledge of contraceptive pills on which patients rely. In addition, further studies with the use of the study protocol described above will allow to determine the most common drug-related problems for different groups of patients and help avoid them by dissemination of necessary knowledge among healthcare professionals and COC users.

\section{State of art}

During the pilot study, 53 potential drug-drug interactions were found. In order to check which ones can affect the COC effectiveness and determine the risk of its clinical importance, a literature review was performed.

\section{Antibiotics}

Our study reported patients' use of macrolide antibiotics (spiramycin, clarithromycin, azithromycin) and fluoroquinolones (norfloxacin). The literature contains a lot of controversy about the impact of antibiotics on the effectiveness of combined hormonal contraception. The data provided by Dinger et al. showed that among 1634 women who had an unplanned pregnancy while taking oral contraceptives, every fifth case reported a concomitant use of antibiotics [14]. However, it should be noted that another study, conducted on a group of 578 patients, showed a similar number of contraceptive failures in a group (a) of only women taking oral contraceptives, and (b) receiving concomitantly COCs and antibiotics [30].

\section{Macrolides}

Recently conducted studies showed no increase in contraceptive failures in connection with the concomitant use of COCs and macrolide drugs (including clarithromycin and azithromycin) [30-34]. These drugs do not cause a decrease in the effectiveness of a COC compound or even raise the concentration of hormones in blood [10]. Although one case of pregnancy during a $\mathrm{COC}$ and minocycline use was documented, the authors concluded that the risk of unintended pregnancy is small but still real [35]. One case of pregnancy have been reported in a COC user who concomitantly received therapy with spiramycin [36].

\section{Fluoroquinolones}

Studies on the effects of fluoroquinolone use on low-dose contraceptives showed no change in the con- centration of ethinyl estradiol (EE) in blood, neither affecting the effectiveness of the drug. The results for the group treated with antibiotic therapy and placebo were comparable and showed clinically insignificant differences [37-40]. No studies investigating the concomitant use of COCs and norfloxacin were found.

Given the above, the study shows that there are no systemic interactions of macrolides and fluoroquinolones with oral hormonal contraceptives, or lower levels of $\mathrm{EE}$ or progesterone in blood. According to the latest guidelines of the World Health Organization (WHO), additional contraceptive methods are not required in case of a concomitant use of combined hormonal contraception with non-liver enzyme-inducing antibiotics [15]. However, the bioavailability of EE as a component of COCs can be lower for some women, which may be due to: elevated steroid hormone metabolism in the liver and intestinal mucosa, changes in the enterohepatic circulation, a borderline low EE level in blood and/or special sensitivity of the intestinal flora to antibiotics [10-13]. This situation, as well as occurrence of adverse effects of antibiotic use - diarrhea and vomiting, may reduce the effectiveness of oral contraceptives, and requires special care $[10,16]$.

\section{St. John's wort}

One case of the use of a formulation containing St. John's wort extract was reported. St. John's wort (Hypericum perforatum L.) is a herbal remedy used mainly to reduce the symptoms of mild depression. Studies and case reports conducted so far showed a decrease in the half-life of $\mathrm{EE}$, and thus a significant reduction in the serum level of contraceptive hormones during concomitant therapy with St. John's wort and COCs. A higher number of breakthrough bleedings during the menstrual cycle, changes in the time of bleeding, and unintended pregnancies were observed among volunteers [18-22, 41]. Researchers agree that St. John's wort extract can affect liver enzymes involved in the metabolism of contraceptive hormones, which leads to a decrease in the serum concentration of COC components and a significant reduction in contraceptive effectiveness [18-22, 41]. Analyzes conducted on patients receiving low doses of St. John's wort extract (500 mg per day) showed no changes in the pharmacokinetics of EE and progestogens, but the study was limited by its small size [17]. Noteworthy, even though Pfrunde et al. showed impact of St. John's wort only on the progestogen component, they observed an increased number of breakthrough bleedings which may result in a higher risk of unintended pregnancies [42]. 
St John's wort, as an inducer of liver enzymes, exhibits a high degree of interaction with other drugs. According to the British Commission for the Safety of Medicines (CSM) and the Clinical Effectiveness Unit (CEU), women taking oral hormonal contraceptives should avoid the concomitant use of COCs and products containing Hypericum perforatum, and, if necessary, use it with great caution [43].

\section{Laxatives}

Anthranoids

Herbal laxative medicines containing anthranoids (Sennae folium, Aloe capensis, Frangulae cortex) irritate nerves and thus stimulate peristaltic movements of the colon [24]. Recent evidence suggests that the use of anthranoid laxatives reduces absorption of $\mathrm{EE}$ and decreases the time of detectable COC levels in blood [23, 24].

Our study reported 3 cases of potential interactions with products containing anthranoids. As its pharmacological effect can occur for four hours after taking a laxative preparation, an interval between taking laxatives and COCs is recommended. If not, it is reckoned that a COC pill may not be absorbed completely [44].

\section{Dietary fiber (Glucomannan)}

Glucomannan is widely used in medicine and patients' diet, however, it may modify properties of orally administered drugs. Studies on rabbits showed that intake of dietary fiber leads to a decrease in EE absorption and may result in ineffective $\mathrm{EE}$ concentrations in blood. However, it also demonstrated some beneficial effects of dosage form (enteric capsules) on EE bioavailability [26, 27]. Another trial proved a reduction of EE enterohepatic recycling in women living on a high-fiber diet [25].

Four cases of the use of a formulation containing dietary fiber was reported. It is recommended to administer COCs 2 hours after or 1.5 hour before consumption of dietary fiber [45].

\section{Adsorbing drugs}

Activated charcoal (Carbo medicinalis), as an adsorbent, inhibits estrogen conjugates' absorption which leads to interruption of EE enterohepatic circulation. As a consequence of the above, a decrease in its concentration in blood and lower effectiveness of COCs can be observed [28].

One case of the concomitant use of Carbo medicinalis and a $\operatorname{COC}$ was noted in the study. A two-hour delay is recommended between the use of activated charcoal and taking a contraceptive pill [28].

\section{Perspectives}

The pilot study confirmed that the designed tool can be effective for identification of some potential drug-related problems. It can be seen that analysis of even a small number of drug lists collected with the use of questionnaire $B$ can provide information about the presence of a potential 'COC-other drug' interaction. Further studies on a higher number of respondents are needed to ensure high-quality statistical data and assess the scope of the problem and to list the most frequent potential interactions.

\section{Acknowledgment}

This project was funded by a research grant of the Poznan University of Medical Sciences (no. 502-05-0331442950740).

\section{Conflict of Interest}

None

\section{References}

1. United Nations Department of Economic and Social Affairs Population Division. World contraceptive Patterns 2013. [Internet] 2014 [cited 2014 Feb 16]; Available from: http://www.un.org/en/development/desa/population/publications/pdf/family/worldContraceptivePatternsWallChart2013.pdf

2. Medart ML. Hormonal contraception and risk of breast cancer. Antykoncepcja - aktualności. 2005;5:7-11.

3. Polish Gynecological Society's recommendations regarding contraception. Ginekol Dypl. 2006:27-31.

4. Erkkola R. Recent advances in hormonal contraception. Curr Opin Obstet Gynecol. 2007;19:547-553.

5. Serfaty D, Wildemeersch D, Verougstraete A, Creatsas G. European Society of Contraception oral contraceptives survey update: birth control methods in "Europe of the 12". Int J Fertil Menopausal Stud. 1995;40(Suppl. 2):73-79.

6. Pearl R. Factors in Human Fertility and their statistical evaluation. Lancet. 1933;225:607-611.

7. Trussel J. Understanding contraceptive failure. Best Pract Res Clin Obstet Gynaecol. 2009;23(2):199-209.

8. Back DJ, Orme ML. Pharmacokinetic drug interactions with oral contraceptives. Clin Pharmacokinet. 1990;18(6):472-484.

9. Al-Busaidi ZQ. Qualitative research and its uses in health care. Sultan Qaboos Univ Med J. 2008;8(1):11-19.

10. Dickinson BD, Altman RD, Nielsen NH, Sterling ML. Drug interactions between oral contraceptives and antibiotics. Obstet Gynecol. 2001;98:853-860.

11. Fotherby K. Interactions with oral contraceptives. Am J Obstet Gynecol. 1990;163:2153-2159.

12. Archer JS, Archer DF. Oral contraceptive efficacy and antibiotic interaction: a myth debunked. J Am Acad Dermatol. 2002:46:917-923. 
13. DRossi SS, Hersh EV. Antibiotics and oral contraceptives. Dent Clin North Am. 2002;46:653-664.

14. Dinger J, Minh TD, Buttmann N, Bardenheuer K. Effectiveness of Oral Contraceptive Pills in a Large US. Cohort Comparing Progestogenand Regimen. Obstet Gynecol. 2011;117:33-40.

15. World Health Organization. Medical Eligibility Criteria for Contraceptive Use ( $3^{\text {rd }}$ edn) 2010. [Internet] 2013 [cited 2013 July 8]; Available from: http:// www.who.int/reproductivehealth/publications/family _planning/9789241563888/en/index.html.

16. Official Polish Gynecological Society Expert Panel position on reasons for hormonal contraceptive failure. Ginekol Pol. 2011;82:313-317.

17. Will-Shahab L, Bauer S, Kunter U, Roots I, Brattström A. St John's wort extract (Ze 117) does not alter the pharmacokinetics of a low-dose oral contraceptive. Eur J Clin Pharmacol. 2009;65:287-294.

18. Schwarz UI, Büschel B, KirchWSchwarz UI, Büschel B, Kirch W. Unwanted pregnancy on self-medication with St John's wort despite hormonal contraception. Br J Clin Pharmacol. 2003;55:112-113.

19. Murphy PA. St. John's Wort and oral contraceptives: reasons for concern? J Midwifery Womens Health. 2002;47:447-450.

20. Hall SD, Wang Z, Huang SM, Hamman MA, Vasavada N, et al. The interaction between St John's wort and an oral contraceptive. Clin Pharmacol Ther. 2003;74:525-535.

21. Murphy PA, Kern SE, Stanczyk FZ, Westhoff CL. Interaction of St. John's wort with oral contraceptives: effects on the pharmacokinetics of norethindrone and ethinyl estradiol, ovarian activity and breakthrough bleeding. Contraception. 2005;71:402-408.

22. Yue QY, Bergquist C, Gerdén B. Safety of St John's wort (Hypericum perforatum). Lancet. 2000;355:576-577.

23. Lewis SJ, Oakey RE, Heaton KW. Intestinal absorption of oestrogen: the effect of altering transit-time. Eur J Gastroenterol Hepatol. 1998;10:33-39.

24. Lewis SJ, Heaton KW, Oakey RE, MGarrigle HH. Lower serum oestrogen concentrations associated with faster intestinal transit. Br J Cancer. 1997;76(3):395-400.

25. Sher A, Rahman A. Role of diet on the enterohepatic recycling of estrogen in women taking contraceptive pills. J Pak Med Assoc. 1994;44:213-215.

26. González A, Fernández N, Sahagún A, García JJ, Diez MJ, Castro LJ, et al. Effect of glucomannan and the dosage form on ethinylestradiol oral absorption in rabbits. Contraception. 2004;70:423-427.

27. Fernández N, Diez MJ, Terán MT, García JJ, Calle AP, Sierra M. Influence of Two Commercial Fibers in the Pharmacokinetics of Ethinylestradiol in Rabbits. J Pharmacol Exp Ther. 1998;286:870-874.

28. Carbo Medicinalis VP, Polfa Rzeszów: Medycyna Praktyczna; 2009. [Internet] 2013 [cited 2013 July 1]; Available from: http://www.medonet.pl/leki,profil-leku,1591519,1,carbo-medicinalis-vp,index.html

29. FRHGuidance (January 2011) Drug Interactions with Hormonal Contraception. Faculty of Sexual and Reproductive Healthcare Clinical Effectiveness Unit. [Internet] 2013 [cited 2013 June 28]; Available from: www.fsrh.org/pdfs/ CUguidancedruginteractionshormonal.pdf
30. Helms SE, Bredle DL, Zajic J, Jarjoura D, Brodell RT, Krishnarao I. Oral contraceptive failure rates and oral antibiotics. J Am Acad Dermatol. 1997;36:705-710.

31. London BM, Lookingbill DP. Frequency of pregnancy in acne patients taking oral antibiotics and oral contraceptives. Arch Dermatol. 1994;130:392-393.

32. Hughes BR, Cunliffe WJ. Interactions between the oral contraceptive pill and antibiotics. Br J Dermatol. 1990; 122:717-718.

33. Hopkins S. Clinical toleration and safety of azithromycin. Am J Med. 1991;Suppl 3A:4-5.

34. Back DJ, Tjia C, Martin C, Miller E, Salmon P. The interaction between clarithromycin and combined oral contraceptive steroids. J Pharm Med. 1991;2:81-87.

35. de Groot AC, Eshuis H, Stricker BH. [Inefficacy of oral contraception during use of minocycline]. Ned Tijdschr Geneeskd. 1990;134(25):1227-1229.

36. Pedretti E, Brunenghi GM, Morali GC. Interazione tra antibiotici e contraccettivi orali: la spiramicina. Quad Clin Ostet Ginecol. 1991;46:153-4.

37. Maggiolo F, Puricelli G, Dottorini M, Caprioli S, Bianchi W, Suter $F$. The effect of ciprofloxacin on oral contraceptive steroid treatment. Drugs Exp Clin Res. 1991;17:451-454.

38. Csemiczky G, Alvendal C, Landgren BM. Risk for ovulation in women taking a low-dose oral contraceptive (Microgynon) when receiving antibacterial treatment with a fluoroquinolone (ofloxacin). Adv Contracept. 1996;12:101-109.

39. Back DJ, Tjia J, Martin C, Millar E, Mant T, Morrison P, et al. The lack of interaction between temafloxacin and combined oral contraceptive steroids. Contraception. 1991;43:317-323.

40. Scholten PC, Droppert RM, Zwinkels MG, Moesker HL, Nauta JJ, Hoepelman IM. No interaction between ciprofloxacin and an oral contraceptive. Antimicrob Agents Chemother. 1998;42:3266-3268.

41. Ernst E. Second thoughts about safety of St. John's Wort. Lancet. 1999;354:2014-2015.

42. Pfrunder A, Schiesser M, Gerber S, Haschke M, Bitzer J, Drewe J. Interaction of St John's wort with low-dose oral contraceptive therapy: a randomized controlled trial. $\mathrm{Br}$ J Clin Pharmacol. 2003;56:683-690.

43. Chief Medical Officer (CO). Fact Sheet for Health Care Professionals. Interactions between St John's Wort (Hypericum perforatum) preparations. CM/CO/2000/4. London, UK: Department of Health; 2000 [cited 2013 July 3]; Available from: http://open.gov.uk-mca/mcahome.htm.

44. Harmonet - Summary of Product Characteristics, Pfizer Europe.

45. Jarosz M, Dzieniszewski J. Drug-food interctions - an important clinical problem. Służ Zdr. 2000:78-79.

Correspondence address:

Cerbin-Koczorowska Magdalena Department of Pharmaceutical Technology Pharmacy Practice Division Poznan University of Medical Sciences, Poland 70 Bukowska Street, 60-812 Poznan Phone: +48 618547206 Fax: +48 618547208 email: mcerbin@ump.edu.pl 